\title{
Gravitational Radiation and Neutrinos
}

\author{
J. B. Griffiths \\ Department of Mathematics, University of Technology, \\ Loughborough, Leicestershire, England
}

Received May 5, 1972

\begin{abstract}
A class of neutrino-gravitational fields with zero energy-momentum tensor is defined. These space-times may also be interpreted as describing gravitational waves and are of Petrov type $D$ or $N$. A wave-like example of the latter is given.
\end{abstract}

\section{Introduction}

A neutrino field in general relativity may be defined by a spinor $\xi_{A}$ which satisfies the generalised neutrino equation

$$
\sigma^{\mu A \dot{B}} \xi_{A ; \mu}=0
$$

and satisfies Einstein's gravitational equations for an energy-momentum tensor defined by

$$
E_{\mu \nu}=\frac{i}{4}\left\{\sigma_{\mu A \dot{B}}\left(\xi^{A} \xi_{; \nu}^{\dot{B}}-\xi^{\dot{B}} \xi_{; \nu}^{A}\right)+\sigma_{v A \dot{B}}\left(\xi^{A} \xi_{; \mu}^{\dot{B}}-\xi^{\dot{B}} \xi_{; \mu}^{A}\right)\right\} .
$$

(For details of notation see Ref. [1].)

There will, of course, be a class of spinors $\xi_{A}$ which satisfy (1) and for which the energy-momentum tensor defined by (2) will be zero. In some space-times this may be satisfied by the trivial case of $\xi_{A}$ being a constant. However there exists a class of space-times for which non-trivial solutions of $\xi_{A}$ can be found. This class is discussed here. It describes a class of neutrino fields which have zero energy and momentum and hence have no effect upon the gravitational field. Space-time metrics of this class are usually interpreted as gravitational waves. Hence we have a class of gravitational waves which also satisfy the neutrino-gravitational field equations. I shall show that these fields can only be of Petrov type $D$ or $N$.

\section{The Field Equations}

If $\xi_{A}$ is taken as a basis, a second basis spinor $\chi_{A}$ can be defined such that

$$
\xi_{A} \chi^{B}-\chi_{A} \xi^{B}=\delta_{A}{ }^{B}
$$


and a tetrad of null vectors may be defined by

$$
\begin{array}{rlrl}
l_{\mu} & =\xi_{A} \sigma_{\mu}{ }^{A \dot{B}} \xi_{\dot{B}}, & n_{\mu}=\chi_{A} \sigma_{\mu}{ }^{A \dot{B}} \chi_{\dot{B}} \\
m_{\mu} & =\xi_{A}{\sigma_{\mu}{ }^{A \dot{B}} \chi_{\dot{B}},} \quad \bar{m}_{\mu}=\chi_{A} \sigma_{\mu}{ }^{A \dot{B}} \xi_{\dot{B}}
\end{array}
$$

where $l_{\mu}$ may be interpreted as the neutrino flux vector. Now using the method of spin coefficients introduced by Newman and Penrose [2], in their notation equation (1) can be written as

$$
\varepsilon=\varrho, \quad \beta=\tau
$$

and in Eq. (2), $E_{\mu \nu}=0$ implies the further conditions that

$$
\gamma=\bar{\gamma}, \quad \alpha-\bar{\beta}-\bar{\tau}=0, \quad \omega=0, \quad \sigma=0, \quad \kappa=0
$$

where I have put $\varrho=\theta+i \omega$. These conditions may be deduced from a previous paper [3]. It may be seen that $l_{\mu}$ defines a twist-free and shear-free null geodesic congruence.

In order to determine the Riemann tensor for these fields, we may substitute the conditions (3) and (4) into the Ricci identities given by Newman and Penrose. These become

$$
\begin{aligned}
D \theta & =3 \theta^{2} \\
\Psi_{0} & =0 \\
D \tau & =(\tau+\bar{\pi}) \theta+\Psi_{1} \\
2 D \bar{\tau}-\bar{\delta} \theta & =-\bar{\tau} \theta+2 \pi \theta \\
D \tau-\delta \theta & =-2 \tau \theta+\bar{\pi} \theta+\Psi_{1} \\
D \gamma-\Delta \theta & =3 \tau \bar{\tau}+2 \bar{\pi} \bar{\tau}+2 \pi \tau-4 \theta \gamma+\Psi_{2} \\
D \lambda-\bar{\delta} \pi & =-\theta \lambda+\pi^{2}+\bar{\tau} \pi \\
D \mu-\delta \pi & =-\theta \mu+\pi \bar{\pi}-\tau \pi+\Psi_{2} \\
D v-\Delta \pi & =(\pi+\bar{\tau}) \mu+(\bar{\pi}+\tau) \lambda-4 \theta v+\Psi_{3} \\
\Delta \lambda-\bar{\delta} v & =-(\mu+\bar{\mu}) \lambda-2 \gamma \lambda+(6 \bar{\tau}+\pi) v-\Psi_{4} \\
\delta \theta & =3 \theta \tau-\Psi_{1} \\
2 \delta \bar{\tau}-\bar{\delta} \tau & =2 \mu \theta-\bar{\mu} \theta+\tau \bar{\tau}-\Psi_{2} \\
\delta \lambda-\bar{\delta} \mu & =(\mu-\bar{\mu}) \pi+3 \bar{\tau} \mu-\tau \lambda-\Psi_{3} \\
\delta v-\Delta \mu & =\mu^{2}+\lambda \bar{\lambda}+2 \gamma \mu-\bar{v} \pi-4 \tau v \\
\delta \gamma-\Delta \tau & =-2 \tau \gamma+2 \mu \tau-\theta \bar{v}+2 \bar{\tau} \bar{\lambda} \\
\delta \tau & =\bar{\lambda} \theta \\
\Delta \theta-\bar{\delta} \tau & =-\theta \bar{\mu}-2 \tau \bar{\tau}+2 \theta \gamma-\Psi_{2} \\
2 \Delta \bar{\tau}-\bar{\delta} \gamma & =2 \theta v-2 \tau \lambda+2 \bar{\tau} \bar{\gamma}-2 \bar{\tau} \bar{\mu}-\Psi_{3}
\end{aligned}
$$




$$
\begin{aligned}
& D \Psi_{1}-\bar{\delta} \Psi_{0}=6 \theta \Psi_{1}-(8 \bar{\tau}-\pi) \Psi_{0} \\
& D \Psi_{2}-\bar{\delta} \Psi_{1}=3 \theta \Psi_{2}-(4 \bar{\tau}-2 \pi) \Psi_{1}-\lambda \Psi_{0} \\
& D \Psi_{3}-\bar{\delta} \Psi_{2}=3 \pi \Psi_{2}-2 \lambda \Psi_{1} \\
& D \Psi_{4}-\bar{\delta} \Psi_{3}=-3 \theta \Psi_{4}+(4 \pi+4 \bar{\tau}) \Psi_{3}-3 \lambda \Psi_{2} \\
& \Delta \Psi_{0}-\delta \Psi_{1}=(4 \gamma-\mu) \Psi_{0}-6 \tau \Psi_{1} \\
& \Delta \Psi_{1}-\delta \Psi_{2}=v \Psi_{0}+(2 \gamma-2 \mu) \Psi_{1}-3 \tau \Psi_{2} \\
& \Delta \Psi_{2}-\delta \Psi_{3}=2 v \Psi_{1}-3 \mu \Psi_{2} \\
& \Delta \Psi_{3}-\delta \Psi_{4}=3 v \Psi_{2}-(2 \gamma+4 \mu) \Psi_{3}+3 \tau \Psi_{4}
\end{aligned}
$$

Solutions to these equations fall into two distinct cases according as the coefficient $\theta$ is zero or non-zero. Since $\theta$ is proportional to the expansion of the congruence, these cases correspond to non-expanding and expanding fields respectively.

\section{The Expanding Case: $\theta \neq 0$}

In this case it is possible to make a null rotation

$$
\begin{gathered}
l_{\mu} \rightarrow l_{\mu}, \quad m_{\mu} \rightarrow m_{\mu}+a l_{\mu} \\
n_{\mu} \rightarrow n_{\mu}+a \bar{m}_{\mu}+\bar{a} m_{\mu}+a \bar{a} l_{\mu}
\end{gathered}
$$

and choose $a$ so that $\tau=0$. The Ricci identities now require that

$$
\begin{array}{ll}
\varrho=\theta, & \sigma=\tau=\kappa=0 \\
\varepsilon=\theta, \quad \alpha=\beta=0, & \gamma=\bar{\gamma} \\
\mu=\bar{\mu}, & \lambda=v=\pi=0
\end{array}
$$

with the differential conditions

$$
\begin{array}{lll}
D \theta=3 \theta^{2}, & \Delta \theta=2 \theta \gamma-2 \theta \mu, & \delta \theta=0 \\
D \gamma=-\theta \mu-2 \theta \gamma, & & \delta \gamma=0 \\
D \mu=0, & \Delta \mu=-2 \gamma \mu-\mu^{2}, & \delta \mu=0
\end{array}
$$

and the components of the Weyl tensor defined by

$$
\Psi_{0}=\Psi_{1}=\Psi_{3}=\Psi_{4}=0, \quad \Psi_{2}=\mu \theta
$$

Hence it may be seen that these fields are of Petrov type $D$ with propagation vectors $l_{\mu}$ and $n_{\mu}$. 


\section{The Non-Expanding Case: $\theta=0$}

In this case the Ricci identities immediately require that

$$
\begin{aligned}
& \varrho=\sigma=\tau=\kappa=0 \\
& \varepsilon=\alpha=\beta=0, \quad \gamma=\bar{\gamma} \\
& \lambda, \mu, \nu, \pi \quad \text { unrestricted }
\end{aligned}
$$

with the differential conditions

$$
\begin{aligned}
D \gamma & =0 & \delta \gamma & =0 \\
D \lambda-\bar{\delta} \pi & =\pi^{2} & \Delta \lambda-\bar{\delta} v & =-(\mu+\bar{\mu}) \lambda-2 \gamma \lambda+\pi v-\Psi_{4} \\
D \mu-\delta \pi & =\pi \bar{\pi} & \delta \lambda-\bar{\delta} \mu & =(\mu-\bar{\mu}) \pi \\
D v-\Delta \pi & =\pi \mu+\bar{\pi} \lambda & \delta v-\Delta \mu & =\mu^{2}+\lambda \bar{\lambda}+2 \gamma \mu-\bar{v} \pi
\end{aligned}
$$

and the components of the Weyl tensor

$$
\Psi_{0}=\Psi_{1}=\Psi_{2}=\Psi_{3}=0
$$

and $\Psi_{4}$ restricted by

$$
D \Psi_{4}=0, \quad \delta \Psi_{4}=0 .
$$

Hence it may be seen that these fields are of Petrov type $N$ with propagation vector $l_{\mu}$. The greater ambiguity in this case is explained since we still have the freedom of making an arbitrary null rotation on the tetrad.

\section{Examples}

Examples of metrics of this class can readily be obtained from known neutrino-gravitational fields, simply by putting the neutrino energymomentum tensor equal to zero. One such example has already been given [4] for the case of the expanding field. However in most of these cases the neutrino spinor loses its wave-like nature. The following example however retains its wave-like properties.

Consider the metric

$$
g_{\mu \nu}=\left(\begin{array}{rrrr}
0 & 1 & 0 & 0 \\
1 & h_{1} & h_{2} & h_{3} \\
0 & h_{2} & -1 & 0 \\
0 & h_{3} & 0 & -1
\end{array}\right)
$$


where $h_{1}, h_{2}, h_{3}$ are independent of $x^{0}$. Following a previous paper [1], I shall relabel the coordinates

$$
x^{0}=v, \quad x^{1}=u, \quad x^{2}=x, \quad x^{3}=y .
$$

This metric will admit a neutrino field with zero energy-momentum tensor if

$$
h_{1,22}+h_{1,33}-2\left(h_{2,21}+h_{3,31}\right)+\left(h_{3,2}-h_{2,3}\right)^{2}=0
$$

where $\left(h_{3,2}-h_{2,3}\right)$ is a function of $u$ only and if the neutrino field defined by

satisfies

$$
\xi_{A}=h_{1}^{-\frac{1}{4}} e^{l(u)+i m(u)}\left(\delta_{A}^{1}-\delta_{A}^{2}\right), \quad l_{\mu}=\sqrt{2} e^{2 l(u)} \delta_{\mu}^{1}
$$

$$
2 \frac{d m}{d u}=h_{3,2}-h_{2,3} .
$$

One simple solution of these equations may be taken to be

$$
\begin{aligned}
& h_{1}=a^{2}-\left(x^{2}+y^{2}\right) \\
& h_{2}=-y, \quad h_{3}=x
\end{aligned}
$$

where $a$ is a constant. Then

$$
\xi_{A}=h_{1}^{-\frac{1}{4}} e^{l(u)} e^{i u}\left(\delta_{A}^{1}-\delta_{A}^{2}\right), \quad l_{\mu}=\sqrt{2} e^{2 l(u)} \delta_{\mu}^{1}
$$

where $l(u)$ is an arbitrary function. For the cylindrical region $x^{2}+y^{2}<a^{2}$ this defines neutrino or gravitational radiation in the direction of $l_{\mu}$. Because of this choice of $h_{3,2}-h_{2,3}=2$, the wave-like character of $\xi_{A}$ is clearly demonstrated. Thus it may reasonably be interpreted as neutrinos with zero energy and momentum.

Acknowledgements. I am much indebted to Dr. R. A. Newing for his comments on an earlier draft of this paper.

\section{References}

1. Griffiths, J. B.: Int. J. Theoret. Phys. 5, 141-150 (1972).

2. Newman, E. T., Penrose, R.: J. Math. Phys. 3, 566-578 1962).

3. Griffiths, J. B., Newing, R. A.: J. Phys. A 4, 208-213 (1971).

4. Griffiths, J. B.: Submitted for publication.

J. B. Griffiths

Department of Mathematics

University of Technology

Loughborough, Leicester, U.K. 
